\title{
Oral and Human Microbiome Research
}

\author{
Sung-Kyun Chung ${ }^{\dagger}$ \\ Department of Dental Hygiene, Shinhan Univertsity, Uijeongbu 11644, Korea
}

\begin{abstract}
In the past gut microbiome has been the main focus of microbiome research. Studies about the microbiome inside oral cavities and other organs are underway. Studies about the relationship between noninfectious diseases and periodontal diseases, and the negative effects of harmful oral microbes on systemic health have been published in the recent past. A lot of attention is being paid towards fostering a healthy oral microbial ecosystem. This study aimed to understand the roles and effects of the microbiome inside the human body can potentially help cure various diseases including inflammatory bowel diseases with no known cure such as Crohn's disease, atopic dermatitis, obesity, cancer, diabetes, brain diseases and oral diseases. The present study examined technological trends in the correlation between the human microbiome and diseases in the human body, interactions between the human body's immunity, the metabolic system, and the microbiome, and research trends in other countries. While it has been proven that human microbiome is closely correlated with human diseases, most studies are still in the early stage of trying to compare the composition of microbiomes between health and patient groups. Since the oral environment is a dynamic environment that changes due to not only food intake but also other external factors such as lifestyle, hygiene, and drug intake, it is necessary to continue in-depth research on the microbiome composition characteristics to understand the complex functions of oral microorganisms. Analyzing the oral microbiome using computational technology may aid in disease diagnosis and prevention.
\end{abstract}

Key Words: Gastrointestinal microbiome, Metagenome, Microbiome, Oral

\section{Introduction}

The Human Genome Project was regarded as a breakthrough technology in the 1990s, and people expected that it would provide a solution for a considerable portion of intractable diseases by analyzing all the base pairs in humans ${ }^{1)}$. However, analysis of the database revealed that the human genome was much simpler than expected, and that this data alone is insufficient in explaining the complex biological processes in the human body. Following this, many scientists realized the necessity of studying symbiotic microbes in humans and their genes, and started a second genome project. They made an inventory of microbial genes and genomes in an effort to understand over 1,000 species of human microbiota, their $2 \sim 4$ million genes, and the interaction between humans and these microbes ${ }^{2}$. The vast majority $(95 \%)$ of the micro- biota symbiotic with humans resides in digestive organs including the colon, while a large number of microbes also live in respiratory organs, reproductive organs, oral cavity, and on the skin ${ }^{3)}$. As the microbiome is becoming the central focus in next-generation bio-healthcare, attempts are being made to exploit the microbiome for prevention of diseases and enhancement of human health, rather than simply identifying correlations and causations through experiments. This review summarizes the present knowledge regarding the microbiome and outlines recent microbiome research.

\section{Introduction to the Microbiome}

The microbiome is an ecosystem with humans, which consists of bacteria, viruses, and eukaryotic microbes such as fungi. There are variations between individuals in the 
species that comprise the microbiota. However, the core microbes that play an indispensable role in keeping us healthy, exhibit considerable consistency, despite differences in individual characteristics and environmental conditions. Microbiome research began to gather momentum in the late 2000s when it was called 'next generation genomics', and findings from these studies establish that the microbiome has profound effects on not only human physiology, but also on human health and disease. For instance, the microbiome plays a role in monosaccharide absorption in the small intestine and fat buildup in the liver ${ }^{4,5)}$. In particular, the human microbiome differs between individuals and experiences dynamic changes, depending on factors such as family, race, sex, age, diet, region, and lifestyle. Microbes inhabit our skin, oral cavity, teeth, respiratory organs, and reproductive organs, extract nutrients and energy from the food we eat, and help to repel or suppress pathogens ${ }^{6}$.

Microbiome is a compound made up of the words 'microbiota' and 'genome'. It was first defined in 2001 as "the community of commensal (with no helpful exchange or pathogenesis), mutualistic and pathogenic microorganisms that share our body space" ${ }^{\text {"7) }}$. The 2006 study lead by Dr. Jeffrey Gordon from Washington University, that contends that lean and obese people have different gut microbiota, initiated widespread recognition of the microbiome as an essential element in human health ${ }^{8)}$. In another study by Dr. Jeffrey Gordon, the feces of obese and lean mice were inserted into germ-free mice. Follow-up observations revealed that even with the same feed consumption, mice that had the feces of obese mice inserted into them gained an additional $50 \%$ of body fat in 2 weeks compared to those that received lean mice's feces. They reported that the gut microbiota not only assists in digestion and nutrient absorption but also affects diseases like obesity ${ }^{9}$. Based on these study results, the US launched the Human Microbiome Project (HMP), anticipating that analyses of the human microbiota would provide clues regarding the mysteries surrounding human diseases ${ }^{10)}$. The International Human Microbiome Project (IHMP) was formed in order to analyze and share the genetic information of specific microbes that cause diseases in citizens of each nation, and the project has been engaged in active research since then. Reports from various activities and research show that the microbiome is closely involved in nutrient absorption, regulation of drug metabolism, development of the immune system and brain, and infectious disease prevention ${ }^{11)}$.

\section{The Role of the Human Microbiome}

The human fetus develops in a microbe-free environment, but comes in contact with microbes as it passes through the birth canal during delivery, swallows maternal bacteria during breastfeeding, and via skin contact. Once the microbes settle, they develop into a unique microbiome for the individual human depending on a myriad of factors including the external environment and diet. The human microbiome enables us to digest and absorb nutrients we cannot digest alone, plays an active role in metabolism, and affects our immune system. Thus, its implications are not limited to intractable diseases like cancer or Crohn's diseases, but are directly linked to general human health.

\section{Nutrient absorption}

Not all enzymes needed to digest the food we eat are produced in the human body. Around 4,000 species of microbes in the gut complement the function of enzymes by aiding in nutrient absorption, producing vitamins, and preventing inflammation. One of the roles of microbes is carbohydrate digestion. Gut microbiota break down many parts of proteins, lipids, carbohydrates, and other nutrients from the food before our body can absorb them. The microbial (produced during the digestion of consumed food) and human metabolites interact with other microbes, and play a role in diverse areas including the regulation of the immune system, metabolism, nervous systems, and brain and behavior development. The gut microbiota shows remarkable variations between individuals according to the type of food they consume and their dietary changes. Despite controlling for nutrient uptake, we found an association between gut microbiome composition and nutrient absorption profiles of individuals. Previously differences in nutrient uptake profiles were only attributed to genetic differences ${ }^{12}$. 


\section{Immune system regulation}

The concept of the human immune system, a system protecting our body from external pathogens, is expanding to include its interaction with and regulation of the human microbiome ${ }^{13)}$. Microbiome function and population are regulated by immune cells and antibody activity. The microbiome is important in the development of lymphatic systems, which include the thymus and spleen ${ }^{14)}$. Gut microbiome digestion of carbohydrates produces short-chain fatty acids (SCFA), increases production of interleukin (IL)-10, and reduces production of IL-6 and tumor necrosis factor- $\alpha$. SCFA is a critical fatty acid which affects immune system regulation, vitamin production, and protects the intestinal mucosa ${ }^{6}$. Lack of gut microbiome settlement during the neonatal period hampers SCFA formation and leaves the infant vulnerable to autoimmune diseases, atopic dermatitis, asthma, and allergies ${ }^{14}$. Correlations between immune tolerance and the microbiome in patients with atopic dermatitis have been reported. The reduced diversity in skin Gammaproteobacteria in patients with atopic dermatitis decreases IL-10 production, and the heightened proportion of Actinobacteria in the skin of healthy individuals induces an increased production of IL-10 15 .

\section{Drug metabolism regulation}

The microbiome plays a role in regulation of drug response, breaking down or modifying administered drugs or carcinogens in the metabolism of ingested substances. Through various metabolic and catalytic activities, it protects our body from drugs and carcinogens entering the body ${ }^{16,17)}$. The microbiome influences various metabolic reactions including dehydroxylation, decarboxylation, dealkylation, and deamination, and impacts oxalate metabolism ${ }^{18-20)}$. This indicates that metabolism of many drugs like anticancer and cardiovascular drugs, can be facilitated or inactivated by the microbiome, and different treatment regimens may be needed as the same drug may have different reactions among different patients due to their microbiome differences ${ }^{21}$. By harnessing the regulative capabilities of the microbiome in drug metabolism, we can provide customized prescriptions for patients with specific diseases based on a proper diagnosis of their genome and microbiome.

\section{Prevention of infectious diseases}

The microbiome functions as a barrier that blocks infection from external pathogens, and it is known that the microbiome balance can significantly alter our defensive capacity against pathogens. Microbiome imbalance weakens barrier function and causes mucosal damage in the intestinal tract, causing pathogens, toxins, and antigens in the intestine to enter the bloodstream and stimulate the immune system, thus leading to infections and autoimmune diseases. An excess of fast food and processed food, a Westernized diet, frequent alcohol consumption, and antibiotic use prevalent in urban lifestyles, disrupt gut microbiota diversity and cause gut microbiota dysbiosis, resulting in allergies, atopic dermatitis, and inflammatory diseases in the digestive system. In a study that compared travelers who contracted diarrhea during a trip with those who did not, researchers found that the presence of Lactobacillus ruminis in higher proportions in a person's microbiome was linked with lower diarrhea incidence and severity ${ }^{22)}$.

\section{Microbiome and Diseases}

The human microbiome is an ecosystem consisting of various symbiotic microbes, and the discovery of its relationships with disease and health led to the possibility of using microbes in diagnosing and treating modern diseases without known origins.

\section{Obesity and diabetes}

Over $90 \%$ of bacteria living in the intestines of patients with obesity belong to the Firmicutes phylum, while Bacteroidetes constitute 3\% of the population. Firmicutes and Bacteroidetes are gut microbes related to obesity; Firmicutes causes obesity while Bacteroidetes inhibits it ${ }^{8,23)}$.

Inducing obesity in healthy mice through a high-fat diet changes its microbiome to an obese type, and transplanting the gut microbiome of obese mice into a germ-free mice facilitates fat accumulation in the mouse ${ }^{9,24,25)}$.

Although causative relationships are unclear, there were more Clostridia in the microbiome of patients with type 2 
diabetes than in healthy controls. Other studies found that butyrate-producing bacteria are reduced in patients with type 2 diabetes ${ }^{26)}$. The association between gut microbiota and diabetes is being investigated, and some studies reveal that the microbiota and its metabolites contribute to type 2 diabetes by affecting energy, carbohydrate metabolism, and inflammation ${ }^{27)}$.

\section{Inflammatory bowel disease}

Microbiome composition is a major factor that causes inflammatory bowel disease (IBD), the major types of which are ulcerative colitis and Crohn's disease. Though the disease shows a wide range of symptoms and clinical courses, genetics and gut microbiome are thought to be common causes ${ }^{28)}$. Studies on the relationship between IBD and gut microbiome have found reduced gut microbiota diversity, an increase in Enterobacteriaceae, and a decrease in Firmicutes including Fecalibacterium prauzsnitzii. When comparing patients with Crohn's disease and ulcerative colitis with controls, patients with Crohn's disease show decreased Firmicutes and increased Gammaproteobacteria, Escherichia coli and Mycobacterium paratuberculosis. An Enterobacteriaceae increase was characteristic of ulcerative colitis, and both Crohn's disease and ulcerative colitis patients exhibited a decrease in $F$. prauzsnitzii ${ }^{29)}$. Mutualistic gut microbes were found to be a play a role in increased immunological homeostasis and IBD suppression and treatment. Further research on the exact role of gut microbiota and immune response is expected to advance the development of diagnostic and therapeutic methods for IBD.

\section{Cognitive impairments}

Analysis into the gut microbiota of healthy mice and mice with Alzheimer's disease confirmed that the two groups had different amounts and compositions of gut microbiota, and inserting the gut microbiota of mice with Alzheimer's into a healthy rat caused a rise in beta-amyloid levels, which is considered to be the main causative factor of Alzheimer's. Analyses to identify bacteria associated with Alzheimer's revealed that there were less Firmicutes and Actinobacteria, and Bacteroidetes were more abundant. Based on a twin study report on dementia that linked tooth loss and dementia, the association between oral microbial composition and dementia was hypothesized ${ }^{30)}$. Two mechanisms explaining the association between oral microbiome and the brain, especially in Alzheimer's disease, have been proposed ${ }^{31)}$. First, aging decreases microbiome-stabilizing oral saliva secretion, thus causing harmful microbes to overpopulate. Second, trigeminal and olfactory nerves may serve as a passage for oral microbes to invade the brain or transmit substances such as amyloids secreted by the microbes ${ }^{32}$.

\section{Cardiovascular disease}

Arteriosclerosis is usually caused by plaque formation in the arteries. The plaques harden and eventually clog the arteries. However, some people with arteriosclerosis have no plaque, and toxic metabolites were detected inside their intestines. These toxic metabolites, such as trimethylamine produced by gut microbiota, are suspected to work in a manner similar to plaque ${ }^{33)}$. Cardiovascular risks including heart failure and stroke increase with increase trimethylamine levels. The gut microbiota generates trimethylamine from carnitine and lecithin, causing cardiovascular diseases $^{33)}$. The oral microbiome is also known to affect cardiovascular disease. Together, these results suggest that everyday human microbiome management is important in order to prevent and alleviate a variety of diseases, including cardiovascular diseases.

\section{Global Research Trends}

The microbiome is receiving a lot of attention as a major global research interest in the age of the Fourth Industrial Revolution, following innovations in information and communications technology. The 2007 HMP Jumpstart signaled the propulsion of microbiome research. Early human microbiome research started with simple studies on culture methods. However, it later showed massive progression with an explosion of data and developments in metagenomics. From 2012 onwards, national level studies started taking place in Korea, France, Australia, Japan, and other countries, in addition to existing projects led by the US and the EU (Table 1) ${ }^{34)}$. Research areas expanded to include respiratory organs, skin, oral cavity, and female 
reproductive organs.

\section{International Human Microbiome Consortium}

The International Human Microbiome Consortium (IHMC) was organized in order to analyze and share genetic information from specific disease causing microbes globally. There are currently nine member states, and Korea joined in 2011. Major research programs include the HMP and National Microbiome Initiative (NMI) in the US, MetaGenoPolis (MGP) and International Human Microbiome Standards (IHMS) led by France, the Korean Microbiome Diversity Using Korean Twin Cohort Project by Korea, the Australian Jumpstart HMP supported by the Australian government, Canadian Microbiome Initiative
(CMI) supported by the Canadian government and The Metagenomics of the Human Intestinal Tract (MetaHIT) funded by the European Commission.

\section{Human Microbiome Project}

The US implemented HMP as a 5-year research plan at the federal level, supervised by the National Institutes of Health (NIH), in order to identify and characterize the microbes that affect human health and disease. HMP started with the goal of completing an exhaustive map after analyzing the genome base pairs of approximately 3,000 species of microbes inhabiting five parts of our body, namely the oral cavity, skin, vagina, intestinal tract, and lungs. Although the exact causative relationships are

Table 1. Major International Microbiome Projects

\begin{tabular}{|c|c|c|c|}
\hline Country & Project title & Duration & Objective \\
\hline International & $\begin{array}{l}\text { International Human Microbiome } \\
\text { Consortium (IHMC) }\end{array}$ & $2010 \sim$ & $\begin{array}{l}\text { Set principles and policies to study microbiome. Mediate } \\
\text { projects to generate a comprehensive data resource. }\end{array}$ \\
\hline \multirow[t]{2}{*}{ EU } & $\begin{array}{l}\text { Metagenomics of the Human Intestinal } \\
\text { Tract (MetaHIT) }\end{array}$ & $2008 \sim 2011$ & $\begin{array}{l}\text { Construct large catalog of intestinal microbiome and } \\
\text { genes of IBD and obesity. }\end{array}$ \\
\hline & $\begin{array}{l}\text { International Human Microbiome } \\
\text { Standards (IHMS) }\end{array}$ & $2011 \sim 2015$ & $\begin{array}{l}\text { Establish a standard protocol for human microbiome } \\
\text { studies. }\end{array}$ \\
\hline \multirow[t]{6}{*}{ USA } & NIH Human Microbiome Project (HMP) & $2008 \sim 2015$ & $\begin{array}{l}\text { Generate resources of the human microbiome. Diagnosis } \\
\text { and treatment through a correlation between diseases } \\
\text { and microbiome. }\end{array}$ \\
\hline & Home Microbiome Project & $2012 \sim$ & Analyze bacterial community in living space. \\
\hline & $\begin{array}{l}\text { Data Analysis and Coordination } \\
\text { Center (DACC) }\end{array}$ & $2008 \sim 2013$ & Assist in standard of data pipeline. \\
\hline & Hospital Microbiome Project & $2012 \sim 2014$ & $\begin{array}{l}\text { Analyze bacterial community in hospital, and find } \\
\text { correlation between the bacteria and patient. }\end{array}$ \\
\hline & $\begin{array}{l}\text { Microbiome Quality Control Project } \\
\text { (MBQC) }\end{array}$ & $2013 \sim$ & Establish analytic pipeline of human fecal microbiome. \\
\hline & American Gut Project & $2013 \sim$ & $\begin{array}{l}\text { Study of bacterial diversity of the public and construct } \\
\text { data resource. }\end{array}$ \\
\hline Japan & $\begin{array}{l}\text { Japanese Consortium for Human } \\
\text { Microbiome (JCHM) }\end{array}$ & $2014 \sim$ & $\begin{array}{l}\text { Study of Japanese specific intestinal microbiome and } \\
\text { disease biomarker. }\end{array}$ \\
\hline Canada & Canadian Microbiome Initiative (CMI) & $2009 \sim$ & $\begin{array}{l}\text { Support seven research teams to conduct a prospective } \\
\text { study of diseases by modeling and mapping microbial } \\
\text { diversity. }\end{array}$ \\
\hline \multirow[t]{2}{*}{ France } & MicroObes & $2008 \sim 2010$ & Research on obesity and human intestinal microbiome. \\
\hline & MetaGenoPolis (MGP) & $2012 \sim 2019$ & $\begin{array}{l}\text { Find the correlation of non-infectious diseases and } \\
\text { intestinal microbiome. }\end{array}$ \\
\hline Korea & $\begin{array}{l}\text { Korean Microbiome Diversity Using } \\
\text { Korean Twin Cohort Project }\end{array}$ & $2010 \sim 2015$ & $\begin{array}{l}\text { Study on human diseases related microbiome using twin } \\
\text { cohort and characterize Korean specific microbiome. }\end{array}$ \\
\hline Australia & $\begin{array}{l}\text { The Australian Jumpstart Human } \\
\text { Microbiome Project }\end{array}$ & 2009 & $\begin{array}{l}\text { Analyze metagenome of intestinal microbes and } \\
\text { Australian specific microbiome. }\end{array}$ \\
\hline
\end{tabular}

Adapted from the article of Choi et al. (Allergy Asthma Respir Dis 4: 311-320, 2016) ${ }^{34)}$ in accordance with the Creative Commons Attribution Non-Commercial (CC BY-NC 4.0) license.

IBD: inflammatory bowel disease. 
yet to be revealed, the gut microbiota composition of people with various diseases differs greatly from that of healthy people, and gut microbiota differences between people with autism, mental illnesses, and Alzheimer's and healthy people have been published. The ultimate goal of the HMP is to reveal the association between disease and human microbiome changes, and to identify correlations between the human microbiome and disease and health.

\section{Metagenomics of the Human Intestinal Tract}

The MetaHIT project, that started in 2008 and was led by French researchers, was active until 2012 with participation from 15 institutions in 8 countries (France, Denmark, Germany, Spain, Italy, the Netherlands, the UK, and China). The goal of MetaHIT was to identify the role of the gut microbiome linked with IBD and obesity, and to analyze their correlations. As a result, a reference catalog of microbial genes comprising the gut microbiome was constructed, and a method capable of analyzing gene frequency differences from individual distribution of microbiota was developed.

\section{MetaGenoPolis}

The MGP was founded in order to investigate influences of gut microbiota on noninfectious diseases, and to examine how the gut microbiome affects human health and infectious diseases through interactions between microbes. Human gut microbiome analysis and genome analysis were conducted, bioinformatics tools for sample analysis were developed, and databases were constructed from results of the analysis.

\section{Human MetaGenome Consortium Japan}

In 2004, Japanese researchers discovered a link between gut microbiota and the brain by observing a twofold increase in the release of stress hormone in a mouse after removal of its gut microbiota. This study was the starting point that led to the establishment of the Human MetaGenome Consortium Japan (HMGJ). The project aims to identify distinct gut microbiome characteristics in the Japanese population, and construct a Japanese specific database. It is a large-scale microbiome project which collects, shares, integrates, and manages human and animal microbiome research data in Japan, and corporate and government research institutes are its participants.

\section{Beijing Genome Institute}

China has been conducting a joint microbiome research project with Europe and Japan. With the goal of completing the 10,000 Microbial Genome Project, Beijing Genome Institute (BGI) has been conducting research on various microbes with diverse applications in agriculture and livestock industry, bioenergy, the environment, and medicine since 2009. It currently plans to conduct the Chinese Microbiome Project.

\section{Conclusion}

Microbiome research studies the diverse microbes inside the human body, and microbiome industry is gaining interest as a new growth driver in biotechnology, which is an essential component of the Fourth Industrial Revolution. Expectations are high about the possibility of novel diagnostic strategies and drug development based on the microbiome, as research reveals the microbiome's impact on a number of diseases including bowel diseases, atopic dermatitis, obesity, metabolic diseases, and depression ${ }^{35-39)}$. While microbiome research has been active globally, many studies still simply settle for evidence that microbial imbalance in the body affects a disease. Although microbiome research is yet to be fully established in Korea, the government has been investing on human microbiome studies since 2016 , especially on studies, which can improve therapeutic strategies or health. Predicted marketability is high for microbiomebased medicine, as it can be applied to invent cures not only for autoimmune diseases such as rheumatoid arthritis, but also for gastrointestinal diseases such as Crohn's disease and IBD, diabetes, and so on ${ }^{40-42)}$.

A healthy oral cavity is the home for many ordinary bacteria, fungi, and viruses. Over 600 species are estimated to inhabit the oral cavity, some of which are pathogenic while others are mutualistic. When oral and gut microbiota of a healthy group and a group with metabolic disease were compared in a twin study of oral microbiota in 228 people, the two groups showed a significant difference in 
microbiota diversity ${ }^{43)}$. Neisseria and Granulicatella were more visible in the oral cavity of the group with metabolic diseases, and quantitative investigation using quantitative polymerase chain reaction obtained the same result ${ }^{43)}$. These oral microbes associated with metabolic diseases are expected to serve as biomarkers for predicting drug response, and assist in research about their pathogenesis mechanisms. In 2016 a survey on male and female adults, periodontal disease was found to be significantly correlated with other diseases including angina, cerebral infarction, and cardiovascular diseases such as myocardial infarction, rheumatoid arthritis, diabetes, and osteoporosis ${ }^{44-49}$. Porphyromonas gingivalis, which causes periodontal diseases, can also enter the bloodstream and cause the formation of atheroma ${ }^{50,51)}$. Reports also indicate that it can activate platelets and induce platelet-leukocyte aggregates, causing a prothrombotic state ${ }^{50,51)}$. Prevalence of bacteremia and endotoxemia were higher in patients with periodontitis than patients with gingivitis or healthy people. P. gingivalis, Actinobacillus actinomycetemcomitans and other periodontal pathogens were found in atherosclerotic tissue biopsies from patients with periodontal disease ${ }^{52}$. Most dental diseases such as gingivitis, dental caries, halitosis, and stomatitis can be attributed to the activity of harmful oral bacteria, and recent claims about their effect on systemic health are drawing attention to the need to maintain a healthy oral microbial ecosystem. Therefore, a need for research methods to analyze oral microbiome and a plan for long-term research is increasing.

\section{Notes}

\section{Conflict of interest}

No potential conflict of interest relevant to this article was reported.

\section{Ethical approval}

It is a reviewed paper so no deliberation required.

\section{ORCID}

Sung-Kyun Chung, https://orcid.org/0000-0002-4022-1620

\section{Acknowledgements}

This work was supported by a 2017 research grant from Shinhan University, Republic of Korea.

\section{References}

1. Collins FS, Morgan M, Patrinos A: The Human Genome Project: lessons from large-scale biology. Science 300: 286-290, 2003. https://doi.org/10.1126/science.1084564

2. Relman DA, Falkow S: The meaning and impact of the human genome sequence for microbiology. Trends Microbiol 9: 206-208, 2001. https://doi.org/10.1016/S0966-842X(01)02041-8

3. Grice EA, Segre JA: The human microbiome: our second genome. Annu Rev Genomics Hum Genet 13: 151-170, 2012. https://doi.org/10.1146/annurev-genom-090711-163814

4. Jeffery IB, O'Toole PW: Diet-microbiota interactions and their implications for healthy living. Nutrients 5: 234-252, 2013. https://doi.org/10.3390/nu5010234

5. Krautkramer KA, Kreznar JH, Romano KA, et al.: Diet-microbiota interactions mediate global epigenetic programming in multiple host tissues. Mol Cell 64: 982-992, 2016. https://doi.org/10.1016/j.molcel.2016.10.025

6. Kau AL, Ahern PP, Griffin NW, Goodman AL, Gordon JI: Human nutrition, the gut microbiome and the immune system. Nature 474: 327-336, 2011. https://doi.org/10.1038/nature10213

7. Lederberg J, McCray AT: 'Ome sweet 'omics - a genealogical treasury of words. Scientist 15: 8, 2001.

8. Ley RE, Turnbaugh PJ, Klein S, Gordon JI: Microbial ecology: human gut microbes associated with obesity. Nature 444: 1022-1023, 2006. https://doi.org/10.1038/4441022a

9. Turnbaugh PJ, Ley RE, Mahowald MA, Magrini V, Mardis ER, Gordon JI: An obesity-associated gut microbiome with increased capacity for energy harvest. Nature 444: 1027-1031, 2006. https://doi.org/10.1038/nature05414

10. NIH HMP Working Group, Peterson J, Garges S, et al.: The NIH Human Microbiome Project. Genome Res 19: 2317-2323, 2009. https://doi.org/10.1101/gr.096651.109

11. Human Microbiome Project Consortium: Structure, function and diversity of the healthy human microbiome. Nature 486 : 207-214, 2012. https://doi.org/10.1038/nature11234

12. Bäckhed F, Ding H, Wang T, et al.: The gut microbiota as an 
environmental factor that regulates fat storage. Proc Natl Acad Sci U S A 101: 15718-15723, 2004. https://doi.org/10.1073/pnas.0407076101

13. Round JL, Mazmanian SK: The gut microbiota shapes intestinal immune responses during health and disease. Nat Rev Immunol 9: 313-323, 2009. https://doi.org/10.1038/nri2515

14. Wold AE: The hygiene hypothesis revised: is the rising frequency of allergy due to changes in the intestinal flora? Allergy 53(46 Suppl): 20-25, 1998. https://doi.org/10.1111/j.1398-9995.1998.tb04953.x

15. Hanski I, von Hertzen L, Fyhrquist N, et al.: Environmental biodiversity, human microbiota, and allergy are interrelated. Proc Natl Acad Sci U S A 109: 8334-8339, 2012. https://doi.org/10.1073/pnas.1205624109

16. Carmody RN, Turnbaugh PJ: Host-microbial interactions in the metabolism of therapeutic and diet-derived xenobiotics. J Clin Invest 124: 4173-4181, 2014. https://doi.org/10.1172/JCI72335

17. Johnson CH, Patterson AD, Idle JR, Gonzalez FJ: Xenobiotic metabolomics: major impact on the metabolome. Annu Rev Pharmacol Toxicol 52: 37-56, 2012. https://doi.org/10.1146/annurev-pharmtox-010611-134748

18. Jones ML, Tomaro-Duchesneau C, Prakash S: The gut microbiome, probiotics, bile acids axis, and human health. Trends Microbiol 22: 306-308, 2014. https://doi.org/10.1016/j.tim.2014.04.010

19. Ridlon JM, Kang DJ, Hylemon PB, Bajaj JS: Bile acids and the gut microbiome. Curr Opin Gastroenterol 30: 332-338, 2014. https://doi.org/10.1097/MOG.0000000000000057

20. Sagar NM, Cree IA, Covington JA, Arasaradnam RP: The interplay of the gut microbiome, bile acids, and volatile organic compounds. Gastroenterol Res Pract 2015: 398585, 2015. https://doi.org/10.1155/2015/398585

21. Spanogiannopoulos P, Bess EN, Carmody RN, Turnbaugh PJ: The microbial pharmacists within us: a metagenomic view of xenobiotic metabolism. Nat Rev Microbiol 14: 273-287, 2016. https://doi.org/10.1038/nrmicro.2016.17

22. Pop M, Walker AW, Paulson J, et al.: Diarrhea in young children from low-income countries leads to large-scale alterations in intestinal microbiota composition. Genome Biol 15: R76, 2014. https://doi.org/10.1186/gb-2014-15-6-r76

23. Arumugam M, Raes J, Pelletier E, et al.: Enterotypes of the human gut microbiome. Nature 473: 174-180, 2011.

https://doi.org/10.1038/nature09944

24. Turnbaugh PJ, Bäckhed F, Fulton L, Gordon JI: Diet-induced obesity is linked to marked but reversible alterations in the mouse distal gut microbiome. Cell Host Microbe 3: 213-223, 2008. https://doi.org/10.1016/j.chom.2008.02.015

25. Turnbaugh PJ, Hamady M, Yatsunenko T, et al.: A core gut microbiome in obese and lean twins. Nature 457: 480-484, 2009. https://doi.org/10.1038/nature07540

26. Qin J, Li Y, Cai Z, et al.: A metagenome-wide association study of gut microbiota in type 2 diabetes. Nature 490: 55-60, 2012. https://doi.org/10.1038/nature 11450

27. Udayappan S, Manneras-Holm L, Chaplin-Scott A, et al.: Oral treatment with Eubacterium hallii improves insulin sensitivity in $\mathrm{db} / \mathrm{db}$ mice. NPJ Biofilms Microbiomes 2: 16009, 2016. https://doi.org/10.1038/npjbiofilms.2016.9

28. Wlodarska M, Kostic AD, Xavier RJ: An integrative view of microbiome-host interactions in inflammatory bowel diseases. Cell Host Microbe 17: 577-591, 2015. https://doi.org/10.1016/j.chom.2015.04.008

29. Willing BP, Dicksved J, Halfvarson J, et al.: A pyrosequencing study in twins shows that gastrointestinal microbial profiles vary with inflammatory bowel disease phenotypes. Gastroenterology 139: 1844-1854.e1, 2010. https://doi.org/10.1053/j.gastro.2010.08.049

30. Gatz M, Pedersen NL: Study of Dementia in Swedish Twins. Twin Res Hum Genet 16: 313-316, 2013. https://doi.org/10.1017/thg.2012.68

31. Miklossy J: Alzheimer's disease - a neurospirochetosis. Analysis of the evidence following Koch's and Hill's criteria. J Neuroinflammation 8: 90, 2011. https://doi.org/10.1186/1742-2094-8-90

32. Shoemark DK, Allen SJ: The microbiome and disease: reviewing the links between the oral microbiome, aging, and Alzheimer's disease. J Alzheimers Dis 43: 725-738, 2015. https://doi.org/10.3233/JAD-141170

33. Koeth RA, Wang Z, Levison BS, et al.: Intestinal microbiota metabolism of L-carnitine, a nutrient in red meat, promotes atherosclerosis. Nat Med 19: 576-585, 2013. https://doi.org/10.1038/nm.3145

34. Choi S, Cho SH, Yi H: Human microbiome studies in Korea. Allergy Asthma Respir Dis 4: 311-320, 2016. https://doi.org/10.4168/aard.2016.4.5.311 
35. Sivignon A, Bouckaert J, Bernard J, Gouin SG, Barnich N: The potential of FimH as a novel therapeutic target for the treatment of Crohn's disease. Expert Opin Ther Targets 21: 837-847, 2017.

https://doi.org/10.1080/14728222.2017.1363184

36. Mayer EA, Knight R, Mazmanian SK, Cryan JF, Tillisch K: Gut microbes and the brain: paradigm shift in neuroscience. $\mathrm{J}$ Neurosci 34: 15490-15496, 2014. https://doi.org/10.1523/JNEUROSCI.3299-14.2014

37. Arentsen T, Qian Y, Gkotzis S, et al.: The bacterial peptidoglycan-sensing molecule Pglyrp2 modulates brain development and behavior. Mol Psychiatry 22: 257-266, 2017. https://doi.org/10.1038/mp.2016.182

38. Kim S, Kim H, Yim YS, et al.: Maternal gut bacteria promote neurodevelopmental abnormalities in mouse offspring. Nature 549: 528-532, 2017.

https://doi.org/10.1038/nature23910

39. Caricilli AM, Saad MJ: The role of gut microbiota on insulin resistance. Nutrients 5: 829-851, 2013. https://doi.org/10.3390/nu5030829

40. ClinicalTrials.gov: Study of bacteroides thetaiotaomicron in young people aged 16 to 18 years with stable Crohn's disease. Retrieved March 9, 2019, from https://clinicaltrials.gov/ct2/ show/NCT02704728(2018, June 28).

41. ClinicalTrials.gov: A study to evaluate the safety, tolerability and microbiome dynamics of SER-287 in subjects with mild-to-moderate ulcerative colitis. Retrieved March 9, 2019, from https://clinicaltrials.gov/ct2/show/NCT02618187(2018, August 27).

42. Bui TP, Ritari J, Boeren S, de Waard P, Plugge CM, de Vos WM: Production of butyrate from lysine and the Amadori product fructoselysine by a human gut commensal. Nat Commun 6: 10062, 2015. https://doi.org/10.1038/ncomms10062

43. Si J, Lee C, Ko G: Oral microbiota: microbial biomarkers of metabolic syndrome independent of host genetic factors. Front Cell Infect Microbiol 7: 516, 2017. https://doi.org/10.3389/fcimb.2017.00516

44. Dev YP, Khuller N, Basavaraj P, G S: Rheumatoid arthritis among periodontitis patients in Baddi industrial estate of Himachal Pradesh, India: a cross sectional study. J Clin Diagn Res 7: 2334-2337, 2013. https://doi.org/10.7860/JCDR/2013/6237.3518

45. Khantisopon N, Louthrenoo W, Kasitanon N, et al.: Periodontal disease in Thai patients with rheumatoid arthritis. Int J Rheum Dis 17: 511-518, 2014. https://doi.org/10.1111/1756-185X.12315

46. Grau AJ, Becher H, Ziegler CM, et al.: Periodontal disease as a risk factor for ischemic stroke. Stroke 35: 496-501, 2004. https://doi.org/10.1161/01.STR.0000110789.20526.9D

47. Won $\mathrm{JH}, \mathrm{Ha} \mathrm{MN}$ : An association of periodontitis and diabetes. J Dent Hyg Sci 14: 107-113, 2014.

48. Jung JO: Effects of metabolic syndrome on periodontal diseases in Korean adults. J Dent Hyg Sci 12: 245-252, 2012.

49. Han GS, Bae KH, Lee MJ, Choi JS: Evaluation of periodontal status according to sociodemographic and health behavior characteristics. J Dent Hyg Sci 8: 395-401, 2008.

50. Schenkein HA, Loos BG: Inflammatory mechanisms linking periodontal diseases to cardiovascular diseases. J Clin Periodontol 40 Suppl 14: S51-S69, 2013. http://doi.org/10.1111/jcpe.12060

51. Reyes L, Herrera D, Kozarov E, Roldán S, Progulske-Fox A: Periodontal bacterial invasion and infection: contribution to atherosclerotic pathology. J Clin Periodontol 40 Suppl 14: S30-S50, 2013. https://doi.org/10.1111/jcpe.12079

52. Tonetti MS, Graziani F: The cardiovascular system and oral infections. In: Glick M, ed. The oral-systemic health connection : a guide to patient care. Quintessence Publishing, Hanover Park, pp.139-155, 2014. 